\title{
The use of audit to set up a thrombolysis programme in the accident and emergency department
}

\author{
J M Kendall, S E McCabe
}

\begin{abstract}
Objective-To improve the thrombolysis service offered by Gloucester Royal Hospital, by reducing the "door to needle time" (DTN) to $30 \mathrm{~min}$ (from a median of $110 \mathrm{~min}$ ), and increasing the proportion of patients with acute myocardial infarctions receiving thrombolysis to $70 \%$ (from $58 \%$ ). This would be achieved by moving the thrombolysis programme from the coronary care unit (CCU) to the accident and emergency (A\&E) department.
\end{abstract}

Design-The process of audit was used to identify an area of poor performance, set standards, acquire funding, demonstrate achievement, and subsequently secure recurrent funding.

Setting-Gloucester Royal Hospital.

Subjects-946 patients presenting consecutively to the $A \& E$ department with non-traumatic chest pain between August 1993 and March 1994.

Main outcome measures-DTN, overall delay time, and acute myocardial infarction thrombolysis rate.

Results-946 patients were assessed over the eight month period, of whom 266 $(28 \%)$ had suffered an acute myocardial infarction; $182(68 \%)$ received thrombolysis (compared to $58 \%$ previously $P<0 \cdot 05)$. Median DTN was reduced to $38 \mathrm{~min}$ ( $v 110 \mathrm{~min}$ previously, $P<0.0006$ ). $127(70 \%)$ patients received thrombolysis in the $A \& E$ department, and $55(30 \%)$ in the CCU. Median overall delay time between onset of pain and thrombolysis was $3 \mathrm{~h} 35 \mathrm{~min} ; 70 \%$ of patients received thrombolysis within $6 \mathrm{~h}$ of onset of symptoms and $90 \%$ within $12 \mathrm{~h}$. Re-audit has subsequently shown maintenance of improvement.

Conclusions-An in-house A\&E based thrombolysis programme works in the District General Hospital setting; the process of audit can be used to acquire, and subsequently secure, funding for the project. The key to successful implementation of change is sensible resource allocation into adequate staffing and appropriate education.

\section{(f Accid Emerg Med 1996;13:49-53)}

Key terms: thrombolysis; infarction; accident and emergency department; audit; funding.
Thrombolysis reduces the mortality and morbidity of acute myocardial infarction, ${ }^{1-6}$ and maximum benefit is derived from a minimal delay in the institution of treatment. ${ }^{237}$ Early thrombolysis leads to reperfusion of ischaemically jeopardised myocardium, thereby reducing the total area infarcted. ${ }^{8} 9$

It is the aim of health service providers, both in the community and in hospital, to minimise this delay, and substantial reduction can be achieved by more effective use of emergency services, and faster provision of thrombolysis in accident and emergency (A\&E) departments. ${ }^{10-12}$

With these broad aims in mind, an "in-house" A\&E based thrombolysis programme was set up at Gloucester Royal Hospital (GRH), using audit to acquire initial, and secure recurrent, funding for the project.

This paper presents the use of clinical audit, and shows that an $A \& E$ based thrombolysis fast track can be instituted efficiently with appropriate resource allocation.

\section{Methods}

Audit is crucial to the acquisition of funding for clinical projects; specifically, the demonstration of an area of clinical inadequacy through pre-audit and the subsequent setting of standards are the basis for the application of a grant to implement change to hospital practice. Subsequent demonstration of benefit, through audit of results achieved, is the key to securing recurrent funding for the project.

\section{PRE-AUDIT}

It was recognised in 1992 that the service provided by GRH in terms of the "door-toneedle" (DTN) time for patients with acute myocardial infarction was unacceptable, and a preliminary study was carried out to identify where delays were occurring, between the months of April and June 1993.

Over this time a median DTN time of $110 \mathrm{~min}$ was achieved, with thrombolysis occurring on the coronary care unit (CCU); it was shown that a relatively rapid $A \& E$ transit time (mean $=41 \mathrm{~min}$ ) was followed by considerable delay in patient transport. It was also shown that $58 \%$ of all acute myocardial infarction patients were receiving thrombolysis. 
STANDARDS

Standards were set to significantly reduce the DTN time to $30 \mathrm{~min},{ }^{12}$ and, by improving education and awareness regarding thrombolysis, to increase the thrombolysis rate to $70 \%$ in acute myocardial infarction patients. This was to be achieved by moving thrombolysis away from the CCU and into the A\&E department, and by appropriate educational measures.

ACQUIRING FUNDING

The pre-audit showing an area of poor performance, coupled with a plan for improvement of the service, and appropriate standards for achievement, was used to acquire funding. The local purchaser was approached for funding to implement the necessary changes, and a single grant of $£ 57595$ was given, on the understanding that this would become recurrent if the standards set could be achieved and demonstrated through audit.

IMPLEMENTATION AND RESOURCE ALLOCATION It was appreciated that the key to the implementation of change would be adequate staffing and appropriate education; the majority of the funding was therefore invested in a cardiology nurse specialist (employed from our CCU, to work during office hours for six months in the $A \& E$ department) and in an extra 1.6 whole time equivalent nursing staff. The role of the nurse specialist was predominantly educational, so that when her six month post elapsed, the $A \& E$ nursing staff were comfortable and confident in managing patients with acute myocardial infarction and in the administration of thrombolysis.

Appropriate education of the junior medical staff (casualty officers and on call medical senior house officers) was already in place in the form of tutorials and senior A\&E staff involvement in the assessment of chest pain whenever possible. Algorithms for the administration of thrombolysis were posted in the relevant places in the department for easy reference. The most important factor in maintaining motivation and standards was regular monthly audit and feedback of results, in which junior staff were actively involved.

Expenditure also occurred on non-recurrent items, for example, monitors, pumps, a defibrillator, and so on. A more complete breakdown of expenditure is as follows:

$F$ grade cardiology nurse specialist +1.6 whole time equivalent other grades

Equipment (defibrillator, monitor, pumps etc.)

Total (1993 prices)

THE MEDICAL DIRECTORATE

Achieving the backing of the hospital cardiologist was the key to gaining the approval of the medical directorate; he was approached with the results of the pre-audit (which clearly identified an inadequate service), the standards we had set, and our plans to achieve them. All of the hospital physicians and geriatricians had different thresholds and protocols for the administration of thrombolysis, and no attempt was made to agree a treatment protocol with the entire medical directorate. Nationally accepted criteria for patient selection $^{10-12}$ were agreed with the hospital cardiologist, and the opinion of the medical directorate was canvassed through a questionnaire covering the relative and absolute contraindications to thrombolysis. It was emphasised that, with the backing of the cardiologist, a universal hospital policy would be drawn up from their questionnaire responses, and would be instituted in the A\&E department. This approach allowed the physicians and geriatricians to feel involved in the decision making, but successfully avoided repeated meetings which would almost certainly have been inconclusive in determining policy.

AUDIT

Consecutive patients presenting to the $A \& E$ department of GRH complaining of "chest pain" (all forms of non-traumatic chest pain) during the eight months between August 1993 and April 1994 were included in the audit. There were no age exclusion criteria. Nursing staff filled in an audit form with information concerning the patient's presentation and subsequent management. Information was collected regarding the time of onset of chest pain, presentation, and thrombolysis; if thrombolysis was not given an explanation of the reason was expected.

Conventional inclusion and exclusion criteria for the administration of thrombolysis were used, that is, two out of three of the following ${ }^{13-15}:$ (1) a good history of chest pain $>30$ min and $<24 \mathrm{~h}$ duration; (2) ST elevation or left bundle branch block on ECG; (3) raised urgent cardiac enzymes.

All patients were received, assessed, and, if appropriate, given thrombolysis within the A\&E department. On a practical level, each patient was immediately evaluated by the "thrombolysis nurse" for that day, who did baseline observations and an ECG, and contacted the appropriate member of the medical staff. Assessment by a member of the medical team occurred within $10 \mathrm{~min}$; if this was not possible, the patient was assessed by a member of the A\&E staff. Streptokinase was the thrombolytic agent of first choice, and alteplase (rtPA) was available if indicated.

Indications for alteplase were as follows: previous acute myocardial infarction with history of streptokinase administration, recent streptococcal infection, hypotension, and if it was likely that an invasive procedure was imminent (for example, insertion of a temporary pacing wire). We did not have purchasing agreement to use alteplase as recommended by GUSTO ${ }^{20}$ (for example, for anterior acute myocardial infarction).

If appropriate, thrombolysis was prescribed by the assessing doctor, and instituted without delay by the thrombolysis nurse within the $\mathrm{A} \& \mathrm{E}$ department. A chest $x$ ray was only performed before the administration of 


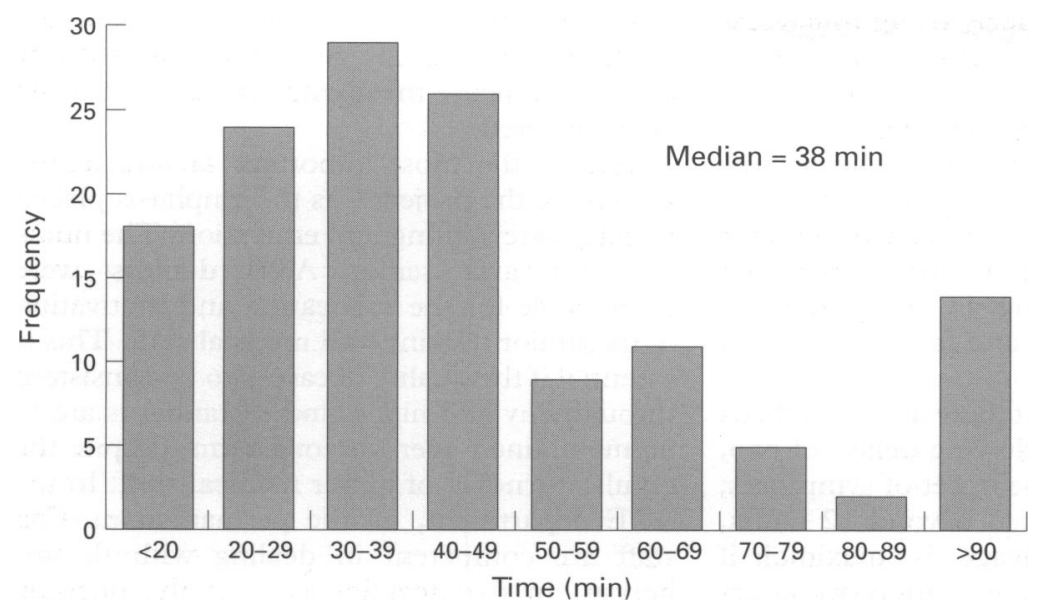

Fig 1 Thrombolysis in the accident and emergency department: "door to needle" time.

thrombolysis if specifically indicated (for example, suspicion of thoracic aneurysm).

The patient, when fully assessed and stable, was then transferred up to the CCU for continued care. All patients had a subsequent analysis of their cardiac enzyme profile, which was taken with their history and ECG to confirm or refute retrospectively a diagnosis of acute myocardial infarction at the time of presentation.

Results were analysed using the Mann Whitney test.

\section{RE-AUDIT}

To ensure that standards are maintained, audit is ongoing, with regular reports of progress (annually) to the purchaser. The results of reaudit performed one year after completion of the main audit project are presented.

\section{Results}

During the audit period of eight months, 946 chest pain assessments were performed; in $266(28 \%)$ of these the patient was shown subsequently to have suffered a myocardial infarction. The remaining patients had angina, chest infections, pulmonary emboli, chest pain of unknown origin, and so on.

During the eight month period, $182(68 \%)$ of patients with acute myocardial infarction received thrombolysis, of whom $127(70 \%)$ received it in the $\mathrm{A} \& \mathrm{E}$ department, and 55

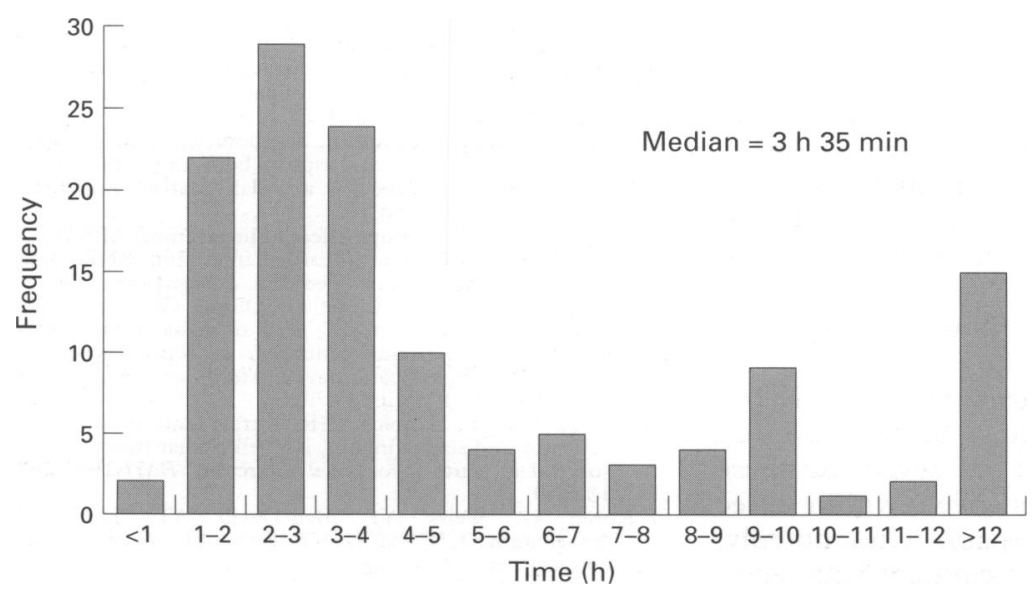

Fig 2 Time from onset of pain to thrombolysis in the accident and emergency department.
Patients with acute myocardial infarction who did not receive thrombolysis $(n=84)$.

\begin{tabular}{lc}
\hline Reason & Number \\
\hline Poor history & 11 \\
Non-diagnostic ECG changes & 17 \\
Duration of pain >24 h & 9 \\
Invasive procedure required & 6 \\
Other cardiac presentation, eg, arrythmia & 13 \\
Hypotension & 3 \\
Active gastrointestinal bleeding & 2 \\
Major surgery within 2 weeks & 1 \\
Thoracic aneurysm & 1 \\
Proliferative diabetic retinopathy & 2 \\
Prolonged CPR & 1 \\
No reason documented & 18 \\
\hline
\end{tabular}

$\mathrm{CPR}$, cardiopulmonary resuscitation.

$(30 \%)$ in the CCU. Patients who did not receive thrombolysis in the $A \& E$ department, but who subsequently received it in CCU, did so because of evolving ECG changes or abnormal cardiac enzyme results, delaying the diagnosis of acute myocardial infarction.

Eighty four patients with acute myocardial infarction $(32 \%)$ did not receive thrombolysis (treatment denial); the reasons documented for these cases are shown in the table. The treatment denial rate in the pre-audit was $48 \%$.

Nineteen patients received thrombolysis who subsequently had no rise in cardiac enzymes, of whom seven $(37 \%)$ received treatment within $3 \mathrm{~h}$ of onset of their pain.

The median A\&E DTN time achieved was $38 \mathrm{~min}$ for the audit period (see fig 1). The median delay between onset of chest pain and thrombolysis was $3 \mathrm{~h} 35 \mathrm{~min}$ (see fig 2).

Re-audit of the month of April 1995 (one year after the audit) showed the following: 67 chest pain assessments, of whom $19(28 \%)$ suffered an acute myocardial infarction; 12 of these $(63 \%)$ received thrombolysis, of whom nine $(75 \%)$ received it in the $A \& E$ department. The median DTN time was $30 \mathrm{~min}$.

\section{Discussion}

The benefits of thrombolysis in acute myocardial infarction are well recognised, as is its administration with minimal delay. We have shown, through audit, a considerable improvement in our management of acute myocardial infarction - in terms of the administration of thrombolysis - by instituting an in-house thrombolysis programme in the $A \& E$ department. A reduction in the DTN median time from 110 minutes to 38 minutes $(P<0.0006$ by Mann Whitney analysis), and an increase in the overall thrombolysis rate from $58 \%$ to $68 \%$ $(P<0.05)$ was achieved.

The numbers in our study are too small to make comments on reduction in morbidity and mortality, and this was not an outcome measure, but it is reasonable to extrapolate from the larger international thrombolysis trials that benefit for patients is being achieved by increasing thrombolysis rate and reducing delay.

It is important to examine the overall time delay between onset of symptoms and thrombolysis when considering the management and outcome of acute myocardial infarction; the DTN time is, of course, a component of this, 
the other aspects of the delay being much less under the influence of the $A \& E$ department, and more under the control of community factors. We recorded a median delay of three hours 35 minutes between onset of symptoms and thrombolysis; with an efficient in-house thrombolysis programme in place, further reduction in this delay will require community based initiatives resulting in better patient recognition of symptoms and faster delivery to hospital.

It is becoming clear that there are watersheds with regard to benefit following delays of two, six, and 12 hours after the onset of symptoms; minimal benefit is achieved beyond 12 hours, whereas myocardial salvage is maximal if thrombolysis can be achieved within two hours of onset of symptoms. ${ }^{1-3} 16$ The majority of our patient population (67\%) presented within the six hour watershed, which underlines the need for an efficient thrombolysis programme: of those patients who received their thrombolysis within the $A \& E$ department, $16 \%$ received it within two hours of onset of their pain, $70 \%$ within six hours, and $90 \%$ within 12 hours.

Thirty two percent of patients with acute myocardial infarction did not receive thrombolysis (see table); if one looks at the reasons for their exclusion, there were, of course, several patients with contraindications to thrombolysis (active gastrointestinal bleeding, recent major surgery, duration of pain $>24$ hours, and so on); the majority were, however, patients who gave a poor history, or who had inadequate ECG changes or a presentation which distracted from an underlying diagnosis of acute myocardial infarction (for example, an arrhythmia, acute cardiac failure, etc). It is disappointing to note that poor documentation also accounted for a number of cases. It is important to remember that when treating a patient with acute cardiac failure or an arrhythmia, the cause may well be an underlying acute myocardial infarct. There is very little one can do to improve a patient's ability to give the perfect history, and consequently the ECG on presentation is probably the most important single determinant for thrombolysis. ${ }^{17}$ It is therefore vital that we select criteria that are maximally specific and sensitive. ST segment elevation is generally accepted to represent acute infarction, and thrombolysis is commonly withheld from patients without ST elevation on the presenting ECG. In the early stages of infarction, ST elevation may be transient and difficult to measure precisely, ${ }^{18}$ and therefore rigidly defined ST elevation is an unsatisfactory precondition for thrombolytic treatment. It has been argued that we should consider thrombolysis in all patients with a convincing history, none of the standard contraindications, and with any abnormality on the ECG. ${ }^{19}$

Nineteen patients received thrombolysis, but did not subsequently show an enzyme rise suggestive of infarction; in seven of these $(37 \%)$ thrombolysis was given within three hours of the onset of pain. Undoubtedly, erroneous diagnosis and treatment will occur, especially when using ECG criteria which are not $100 \%$ specific, but in some cases where treatment was rapidly given, the possibility of an aborted acute myocardial infarction should be considered.

One of the most important factors in the success of the project was the emphasis placed on adequate staffing and education. The nurse specialist and senior A\&E doctors were responsible for the reducation and motivation of the junior nursing and medical staff. This is essential if the quality of care is to be consistent through day and night, and if standards are to be maintained over the long term, despite the regular turnover of junior medical staff. In the A\&E department, all the permanent nursing staff are competent in dealing with thrombolysis; in the hospital as a whole, ongoing tutorials and audit, which reach a wide audience of junior doctors, ensure consistency of practice.

It can be seen that we have not quite achieved the standards we aimed for, but, like all change, there is a learning curve; progress so far has closed the loop in the audit cycle and, following demonstration of the results to date to the health authority purchaser, recurrent funding for the project has been secured.

\section{CONCLUSIONS}

The reduction in morbidity and mortality from acute myocardial infarction is a goal of all health care professionals concerned with the management of ischaemic heart disease. An inhouse thrombolysis programme works in the district general hospital A\&E department; for other departments aiming to establish similar projects, the highlighting of inadequacy by preliminary study, and the subsequent setting of standards, are essential to the acquisition of funding. The keys to its successful implementation are sensible resource allocation for staffing and education. Standards set in any audit exercise should be ambitious but achievable, and completing the audit cycle, by demonstrating that these standards are being achieved or approached, is crucial to securing recurrent funding.

We would like to acknowledge the efforts of the clinical audit department of GRH, and the major nursing contribution of Fran Wilson and Tom Hasnell.

1 Gruppo Italiano Per La Studio Della Streptochinasi Nell'Infarcto Miocardico (GISSI). Effectiveness of intravenous thrombolytic treatment in acute myocardial infarction. Lancet 1987;i:397-402.

2 GISSI. Long term effects of intravenous thrombolysis in acute myocardial infarction: final report of the GISSI study. Lancet 1987;i:871-4.

3 ISIS-2 Collaborative Group. Randomised trial of intra venous streptokinase, oral aspirin, both, or neither amon 17,187 cases of suspected acute myocardial infarction. Lancet 1988; ii:349-60.

4 AIMS Trial Study Group. Effect of intravenous APSAC on mortality after acute myocardial infarction: preliminary report of a placebo controlled trial. Lancet 1988,ii:545-9.

5 Wilcox RG, Von der Lippe G, Ollson CG, Jensen G, Skene AM, Hampton JR. Trial of tissue plasminogen activator for mortality reduction in acute myocardia infarction. Anglo-Scandinavian study of early throm infarction. Anglo-Scandinavian study of early thrombolysis. Lancet 1988;ii:525-30.

6 Van de Werf F, Arnold AGR. Intravenous plasminoge activator and size of infarct, left ventricular function, an survival in acute myocardial infarction. BMF 1988;297: 1374-9.

7 Koren G, Weiss AT, Ben-David AT, Hasin Y, Appelbaum D, Welba S, et al. Prevention of myocardial damage in acute myocardial ischaemia by earlier treatment with intravenous streptokinase. $N$ Engl $7 \mathrm{Med}$ 1988;313:1384-9. 
8 Reimer KA, Lowe JE, Rasmussen MM, Jennings RB. The wavefront phenomenon of ischaemic cell death Circulation 1977;56:786-94.

9 Bergmann SR, Lerch RA, Fox KA, et al. Temporal dependence of beneficial effects of thrombolysis characterised by positron tomography. Am $\mathcal{F}$ Med 1982 ; 73:573-81.

10 Heriot AG, Brecker SJ, Coltert DJ. Delay in presentation after myocardial infarction. $\mathcal{F} R$ Soc Med 1993;86: 642-4.

11 Dalton H, Chappel D, Climie R. Thrombolysis for acute myocardial infarction in a District General Hospital. $\mathcal{f} R$ Soc Med 1989;82:394-5.

12 Birkhead JS. Time delays in provision of thrombolytic treatment in six district hospitals. Joint Audit Committee of the British Cardiac Society and a Cardiology of the British Cardiac Society and a Cardiology Committee of Royal

13 Wilcox RG. Choice of agent: optimal efficacy vs. side effects. In: de Bono D, ed. Thrombolysis: current issues and future directions. Oxford: The Medicine Group (Education), 1992:9-15.
14 Chamberlain DA. Relationship of trials to the general population: who should get thrombolysis and when? In: de Bono D, ed. Thrombolysis: current issues and future directions. Oxford: The Medicine Group (Education) 1992:17-20.

15 Schweitzer P. The electrocardiographic diagnosis of myocardial infarction in the thrombolytic era. Am Heart $\mathcal{f}$ 1990;119:642-54

16 Cobbe SM. Thrombolysis in myocardial infarction; the earlier the better, but how late is too late? $B M F$ 1994; 308:216-7.

17 Timmis A. Early diagnosis of acute myocardial infarction. $B M 7$ 1990;301:941-2.

18 Adams J, Trent R, Rawles J. Earliest electrocardiographic evidence of myocardial infarction: implications for evidence of myocardial infarction: implication

19 Rawles JM. Risk benefit analysis of thrombolytic therapy for acute myocardial infarction: a perspective. Coronary Art acute myocardial infarc

20 The GUSTO Investigators. An international randomised trial comparing four thrombolytic strategies for acute myocardial infarction. $N$ Engl $₹$ Med 1993;329:673-82.

\section{ASSOCIATION OF SURGEONS OF GREAT BRITAIN AND IRELAND ANNUAL MEETING - GLASGOW 22-24 MAY 1996}

The theme will be EVIDENCE BASED MEDICAL PRACTICE and there will be sessions on GUIDELINES as well as a TELEPRESENCE SYMPOSIUM.

Two sessions will be held by the BAEM as follows:-

"The Management of Crime and self-inflicted injury from pre-hospital care to operating theatre"

Joint symposium with the intensive care society and the vascular society: "The Management of the collapsed vascular patient" and "Reception, resuscitation and transfer of the ruptured aortic aneurysm and vascular trauma"

Members of BAEM are welcome to attend this meeting. Further details from Mrs Nechama Lewis, ASGI, tel: 01714056753 fax: 01714309235. 